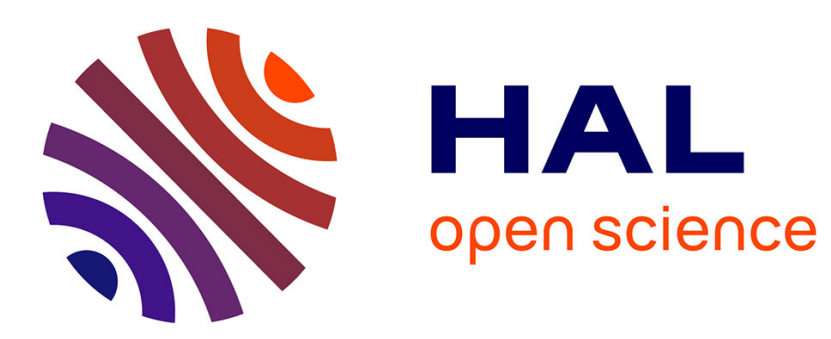

\title{
A Hybrid Approach for RAT Selection in Wireless Heterogeneous Networks
}

\author{
Mohamad Yassin, Marc Ibrahim, Samer Lahoud
}

\section{To cite this version:}

Mohamad Yassin, Marc Ibrahim, Samer Lahoud. A Hybrid Approach for RAT Selection in Wireless Heterogeneous Networks. Third International Conference on Communications and Information Technologies, Jun 2013, Beirut, Lebanon. hal-01110010

\section{HAL Id: hal-01110010 https://hal.science/hal-01110010}

Submitted on 27 Jan 2015

HAL is a multi-disciplinary open access archive for the deposit and dissemination of scientific research documents, whether they are published or not. The documents may come from teaching and research institutions in France or abroad, or from public or private research centers.
L'archive ouverte pluridisciplinaire HAL, est destinée au dépôt et à la diffusion de documents scientifiques de niveau recherche, publiés ou non, émanant des établissements d'enseignement et de recherche français ou étrangers, des laboratoires publics ou privés. 


\section{A Hybrid Approach for RAT Selection in Wireless}

\section{Heterogeneous Networks}

\author{
Mohamad Yassin, Marc Ibrahim \\ Ecole Supérieure d'Ingénieurs de Beyrouth \\ Saint-Joseph University \\ Beirut, Lebanon
}

\author{
Samer Lahoud \\ IRISA \\ University of Rennes 1 \\ Rennes, France
}

\begin{abstract}
Given the diversity and heterogeneity of the existing wireless radio access technologies, a key problem that arises in this context is the radio access network selection. The purpose of radio access network selection is to improve the quality of service experienced by clients and optimize the use of available radio resources. In this context, two approaches can be adopted: either a centralized or a distributed approach. In this paper, based on the two existing radio network selection approaches, we propose a new hybrid approach that combines the distributed one with periodic centralized interventions. We introduce the concept of the proposed hybrid approach, and study its performance. In the hybrid approach, client inbound sessions perform a distributed network selection while the system regularly intervenes to change client associations and/or to modulate the distributed algorithm in order to guarantee an acceptable level of performance. The results show that the hybrid approach performance is close to that of the optimal solution, and is better than that of the distributed approach in specific scenarios. Moreover, the hybrid approach reduces the overall signaling load for the system by decreasing the number of required handovers.
\end{abstract}

Keywords-RAT selection; heterogeneous wireless network; hybrid approach; radio resources

\section{INTRODUCTION}

Nowadays, wireless networks have become very common. A wireless network is based on a radio access technology that provides mobile users the ability to access the network while they are moving, and to use the various available services via a wireless connection.

Wireless networks are experiencing a wide diversification and a large-scale deployment. Indeed, in addition to mobile networks $(2 / 3 / 4 \mathrm{G})$ [1] that are deployed on a large scale there are also many broadband technologies of the IEEE family (IEEE 802.11, IEEE 802.16, etc.)[2].

We can benefit from the coexistence of different technologies [3] to improve the overall system performance, to ensure users' growing demands for resources, to benefit from the available radio resources and to improve the quality of service experienced by clients (in terms of offered bitrates and transmission delays). In this context, a client has the ability to connect to different wireless access networks.
The operator can benefit from this heterogeneity by providing a diversified access via different radio interfaces coexisting in the same geographical area [4].

In this context, two approaches can be adopted: either the centralized optimal approach or the distributed approach. The centralized solution controlled by the operator has the advantage of a better view of the network and the possibility of reaching an optimum set by the operator [5]. The second approach is to let the mobile choose its network based on certain quality measures [6] or based on game theory techniques [7]. This approach is simpler than the previous one, but induces an individual optimality at the expense of an overall network sub-optimality [8].

Several previous researches have studied the problem of radio access network selection. In [9] authors developed and searched to minimize a function that gives the overall system cost. Power optimization in heterogeneous networks is the objective in [10] where authors propose a hybrid power efficient Radio Access Technology (RAT) selection algorithm. In [11] authors have developed and evaluated four distributed heuristic algorithms approaching the optimal solution.

Based on the previous work, we develop in this paper a dynamic model of the hybrid system where we implement the hybrid approach algorithms. In the centralized approach, the system uses a common radio resource management strategy in order to minimize the overall cost function given for all the users ; however, in the distributed approach each client aims at optimizing its own cost function individually, regardless of the other clients and the system state. The distributed approach is an implementation of the 'Always Best Connected ABC' concept [12]. Always Best Connected (ABC) is to select and always connect to the most appropriate network when multiple networks are available.

In this paper, we define a new hybrid approach that lies between the centralized [5] and the distributed [6, 7] approaches. The proposed approach uses a distributed algorithm to associate the client sessions that arrive to the heterogeneous system. Moreover, client associations are periodically adjusted according to a centralized approach. This new approach is studied in different scenarios. The main simulation parameter is the client peak rates perceived from the existing wireless access networks. This paper gives significant 
arguments to answer the question: in which cases and for what scenarios could the hybrid approach be more advantageous than the two existing approaches used separately?

The rest of the paper is organized as follows: the system model and the cost calculation are described in (II). The different approaches for radio access network selection and the new proposed hybrid approach are presented in (III). The simulated scenarios are described in (IV) and the results are reported in (V). Conclusion is given in section (VI).

\section{System Model AND Cost CALCULATION}

The studied geographic area could be simultaneously covered by different types of wireless access networks. These heterogeneous technologies have different characteristics such as coverage, offered bitrates, capacity, etc. The main parameter is the peak rate perceived by the users.

We consider a geographic area entirely covered by a primary radio access technology (i.e., it contains a base station belonging to the primary access technology) and partially covered by a secondary radio access technology (i.e., several access points of the secondary radio access technology). Only clients within the coverage areas of the secondary access points can balance their traffic between the two technologies. Fig. 1 shows a geographic area covered by two radio access technologies with clients that can balance their traffic between the two technologies.

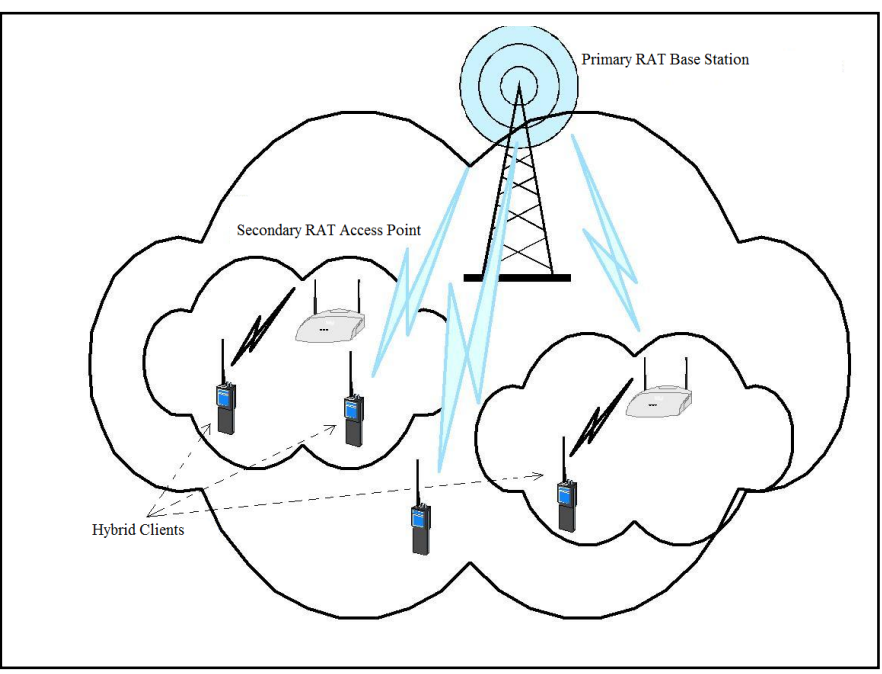

Figure 1. Heterogeneous system with hybrid clients [9]

The model used can be applicable to different types of wireless technologies. In each cell, the peak rate of each user depends on its radio conditions. We adopt a simple model that takes into account client peak rates as the main parameter. We consider only the downlink traffic and we neglect the waiting times (i.e., DIFS and SIFS for Wi-Fi). Therefore, the access scheme (i.e., CSMA/CA for $\mathrm{Wi}-\mathrm{Fi}$ ) leads to a fair rate sharing of the channel among the existing users [11]. All users have the same throughput that is less than the minimum peak rate that a single user can perceive when alone in the cell [13].
We denote by $n$ the number of clients that will be concerned in radio access technology selection. Those users will be indexed by $k=1,2, \ldots n$. We denote by $h(k)$ the secondary technology cell (hotspot) in which the client $k$ exists.

The total cost for a client $k$, defined as the time required for sending a data unit in a heterogeneous environment, will be given by [11]:

$$
C_{k}=T_{k, 0} \cdot\left(1-\theta_{k}\right)+T_{k, h(k)} \cdot \theta_{k}
$$

Given that $T_{k, x}$ is the service time required for sending a data unit in a cell $x$, and $\theta_{k}$ is the fraction of time during which the client $k$ is associated to the access point of the secondary technology $h(k)$.

\section{RAT SELECTION APPROACHES}

\section{A. Existing approaches: description and weakpoints}

In the following, we analyze the centralized and the distributed approaches:

In the centralized approach, the system load balances the downlink traffic of every user between the two radio access technologies in a way to privilege the overall system performance. It gives the optimal solution - the best association of the clients to the existing wireless networks - by minimizing the sum of all the individual cost functions given by equation (1). Client association occurs each time we have a change in the system state: either a client arrival or a departure. The centralized approach requires knowledge of the peak rates of all the users in the system. The main drawback of this approach is the number of handovers to be carried out for each change in the system state (when an arrival or a departure occurs). In fact, the centralized approach will search for the optimal client association profile after each client arrival/departure. It will then modify client association according to the new profile. This will induce an additional number of handovers to achieve the profile modification. These handovers will generate additional signaling traffic that increases network congestion and degrades its performance.

In the distributed approach, each user selfishly strives to improve its own performance regardless of the other users and the system state. It is a simple approach where each client associates individually to the network that offers him the best peak rate. Client association occurs only once, when the client arrives to the system. The distributed approach requires only the knowledge of the peak rates perceived via the two existing radio access technologies. This approach is characterized by a very low processing load (since the network selection is done in a distributed way). In addition, no handover is required because the client decides its association upon his arrival to the system and remains associated to the selected network throughout his session. However, the distributed approach will degrade the performance since it induces a sub-optimality for the overall system: the given solution is not the one with the lowest overall cost. 


\section{B. The new hybrid radio access technology selection approach}

In the centralized approach, the system allocates the downlink traffic between the existing radio access technologies. It balances users' traffic between wireless technologies in order to optimize the total cost. For each change in the network state (client arrival/departure) the system searches for the optimal association profile and associates the clients according to it.

However, in the distributed approach each client aims at minimizing its individual cost function regardless of the other clients and the system state. Hence, each client decides of his association individually when he arrives to the system in order to minimize his own cost.

The hybrid approach consists in adopting the distributed approach, but with periodic system intervention to adjust the client association profile to the existing wireless access networks.

Compared to the centralized approach, the hybrid approach has a lower processing load. In fact, the centralized approach requires a global knowledge of the entire system state and induces a huge processing cost when a change in the system state occurs (arrival or departure). However, hybrid approach interventions occur at regular time intervals (periodic intervention) to adjust the client association to the existing radio access technologies in accordance with the centralized approach.

In addition, intervening periodically to apply the centralized approach may improve the performance of the distributed solution. When the clients choose their association individually the distributed association profile tends to differ from the optimal one since each client aims at choosing the network that suits him the most regardless of other clients and system's state. However, periodic intervention forces the clients to adopt the optimal association profile when it occurs. Therefore, the client association profile will remain close to the optimal association profile.

The hybrid approach works as follows (Fig. 2):

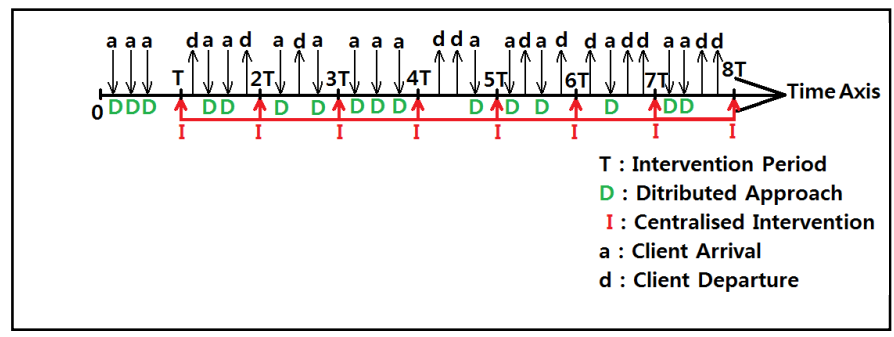

Figure 2. Heterogeneous system adopting the hybrid approach

The hybrid approach performs periodic interventions to adjust the association of clients to the existing networks. When a new arrival occurs (a new client arrives) if the intervention period has not expired, it is the distributed approach that will decide of his association. In addition, if the intervention period has not elapsed and a client leaves our system, the distributed approach does not induce any change in the client association profile.
Consequently, whenever the intervention period elapses (at $T, 2 T, 3 T, \ldots, n T \ldots$ ) we adopt a centralized approach to update the client association profile to the wireless access networks (even if no arrival has occurred since the last intervention). This intervention corrects association errors that may be committed by the distributed decision.

The hybrid approach algorithm must be implemented with a dynamic system model. This algorithm is a compromise between the centralized and the distributed approaches algorithms. In the hybrid approach, several distributed algorithms can be used when adopting the distributed decision: deterministic or probabilistic [11]. The system intervention is performed on a periodic basis to adjust client associations when necessary.

\section{SIMULATED SCENARIOS}

Various scenarios are proposed in order to compare the performance of the different implemented algorithms. For each scenario, we perform a random draw to determine the number of clients that will be located in each of the secondary cells present in the geographic area (one primary and two secondary cells). Then, we perform another random draw weighted by the percentage of clients with higher primary peak rates to determine whether the client has better primary or secondary peak rate. Finally, we perform a random draw to associate each client with a couple of peak rates (primary peak rate, secondary peak rate). These random draws will guarantee a wide variety of distributions during the generation of each scenario. In addition, each scenario is iterated several times, and the average cost is adopted. For each scenario, several trust points are plotted to assess the validity of results.

\section{Simulation Results}

In order to compare the different approaches that can be adopted in radio access technology selection, simulations on several scenarios were performed. All the approaches that have already been mentioned in this paper will be processed and analyzed.

The main parameter for the system model used is client peak rates. IEEE $802.11 \mathrm{~b}$ and $802.11 \mathrm{~g}$ are the two radio access technologies used for the simulation since each one have its own peak rates. The Table 1 shows the main characteristics of Wi-Fi technology (IEEE 802.11b and IEEE 802.11g):

TABLE I. WIFI TECHNOLOGY'S CHARACTERISTICS

\begin{tabular}{|l|c|c|c|}
\hline Technology & Bitrates $\mathbf{( M b} / \mathbf{s})$ & Range (m) & $\begin{array}{c}\text { Frequency } \\
\text { Band } \\
\text { (GHz) }\end{array}$ \\
\hline $802.11 \mathrm{~b}$ & $\begin{array}{c}6,9,12,18,24, \\
36,48,54\end{array}$ & $\begin{array}{c}\text { From } 35 \text { to } \\
100\end{array}$ & 2.4 \\
\hline $802.11 \mathrm{~g}$ & $1,2,5.5,11$ & $\begin{array}{c}\text { From } 25 \text { to } \\
75\end{array}$ & 2.4 \\
\hline
\end{tabular}

To better analyze the simulation results, we define the following ratios (Table II):

TABLE II. CONSIDERED RATIOS 


\begin{tabular}{|c|c|c|c|c|c|}
\hline Ratio 1 & Ratio 2 & Ratio 3 & Ratio 4 & Ratio 5 & Ratio 6 \\
\hline $\mathrm{R} / \mathrm{O}$ & $\mathrm{PR} / \mathrm{O}$ & $\mathrm{PR} / \mathrm{R}$ & $\mathrm{HR} / \mathrm{O}$ & $\mathrm{HPR} / \mathrm{O}$ & $\mathrm{HR} / \mathrm{R}$ \\
\hline
\end{tabular}

Where:

- $\mathrm{R}$ denotes the cost for the distributed deterministic peak rate based algorithm.

- PR denotes the cost for the distributed probabilistic peak rate based algorithm.

- O refers to the cost of the centralized optimal solution.

- HR denotes the cost for the hybrid deterministic peak rate based algorithm.

- HPR denotes the cost for the hybrid probabilistic peak rate based algorithm.

These ratios are the cost ratios of the different algorithms implemented. The cost is the time required by a client to send a data unit via its wireless access network.

If the ratio is greater than 1 , the algorithm in the numerator is more expensive. The solution is more efficient when it has a lower cost. However, when the ratio is less than 1 , the algorithm of the numerator will be the most efficient. It will improve the QoS and the client experience, and it will reduce the total cost (of the system) allowing the operator to make better use of its network, to introduce new services and to take better advantage of the available bandwidth.

\section{A. Cost ratios}

Simulations are performed for scenarios where 500 clients arrive to the system according to a Poisson process of parameter $\lambda(1 \mathrm{client} / \mathrm{s})$. Session durations follow a Pareto distribution, and the system performs periodic interventions with a period $\mathrm{T}=1$ second. The results are reported below (Fig. 3):

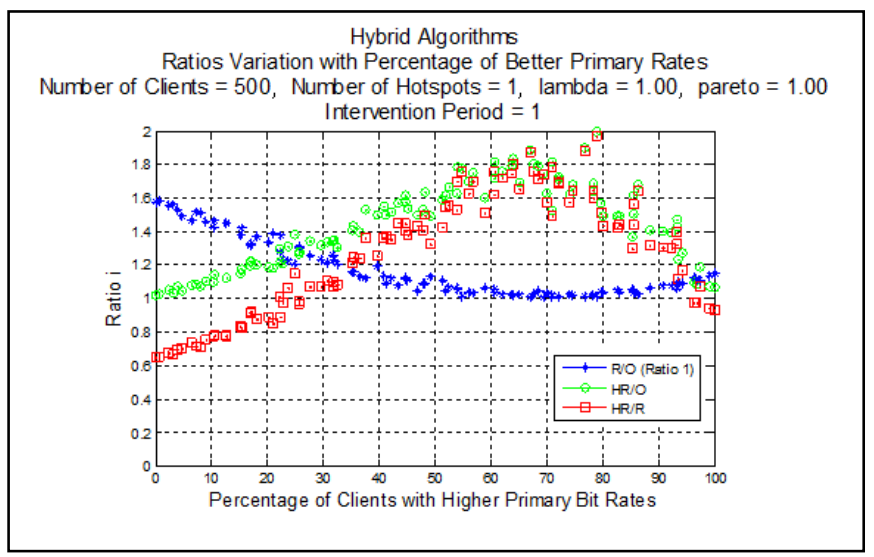

Figure 3. Performance variation depending on clients peak rates distribution

The curves obtained in Fig. 3 show that the distributed deterministic approach's algorithm (without any intervention by the system) leads to a performance that is very close to the centralized approach for equitable client peak rates distribution between the two radio access technologies existing in the coverage area. In fact, the distributed decision is very close to the optimal one, and it will associate the clients to the adequate wireless access network so that we will have the lowest cost.

The hybrid approach's algorithm that performs periodic interventions to adjust client association profile has its performance degraded for equitable distributions of peak rates between the two wireless access networks. In fact, the distributed approach's decision is very close to the centralized decision. Thus, the periodic intervention (to apply the centralized approach's association profile) will adversely affect the performance of the system over time. Therefore, the hybrid solution cost will be greater than that of the centralized approach (and even greater than that of the distributed approach) when the client peak rates distribution is equitable between the two wireless access networks. The hybrid approach's algorithm shows better performance than the distributed one for extreme peak rates distributions since the latter's decision is not close to the optimal one. In this case, the intervention will be interesting since it will adjust the client association profile to the optimal one, which will reduce the cost and improve the performance.

\section{B. Number of handovers}

We also study the number of handovers (HO) that must be performed for each approach. The more we have handovers the more we need signaling and the more we have valuable resources that are consumed. Therefore, we will have fewer resources for the signaling traffic. This will adversely affect the performance of the system.

Simulations (Fig. 4) are made to highlight the cost of each approach in terms of number of handovers:

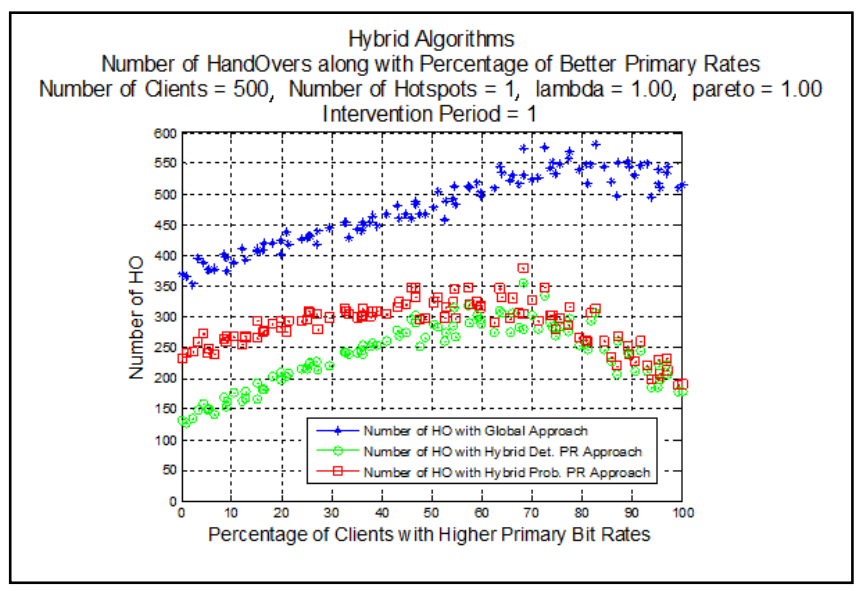

Figure 4. Performance variation depending on clients peak rates distribution

The curves show that the centralized approach has the largest number of handovers. Whereas the number of handovers for the hybrid deterministic or probabilistic peak rate based approach is smaller.

It is important to note that for the distributed approach no handover is required, since the client association to the system is done only once (when the client arrives at the system). The client will always be associated to the chosen network until its session expires. Thus, no handover is done. 
The centralized approach requires a calculation of the best association profile at each change of the system state in order to find the profile with the lowest cost. Thus, the number of handovers made will increase since the association of the clients is modified at each event (arrival/departure).

When the hybrid approach is used, a change in the client association profile will occur for each centralized intervention. Therefore, we will need to perform a number of handovers to modify the client associations. However, the number of required handovers is less than that required for the centralized approach since the association changes does not occur very frequently. The hybrid approach will reduce the number of handovers and the signaling traffic needed to carry them out.

\section{Effect of the intervention period}

Simulations results (Fig. 5) show the influence of the intervention period variation on the hybrid approach performance.

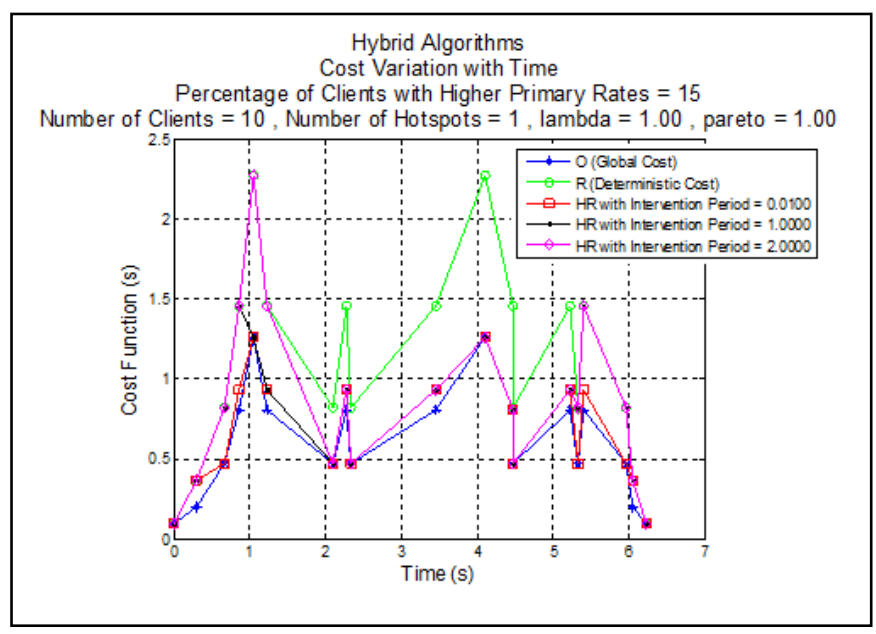

Figure 5. Performance variation in time

These curves (Fig. 5) show that the centralized approach has always the best performance since it provides the lowest cost. The distributed approach is the furthest from the centralized approach. The hybrid approach is a compromise between the two extreme approaches in terms of cost, quality, service and processing load.

The intervention period is the main parameter for the hybrid approach. The more the intervention period is small, the more the interventions are frequent, and the more the hybrid approach is close to the centralized one (optimal). However, this will increase the number of handovers and the signaling load. Furthermore, the cost function increases when the intervention period increases, but the signaling load decreases.

The centralized approach is indeed a special case of the hybrid approach but with an intervention period that tends to zero (or an intervention frequency that tends to infinity). Therefore, when the intervention period decreases (the intervention frequency increases), the hybrid approach's performance will be closer to that of the centralized approach.

\section{CONCLUSION}

In this paper, a hybrid approach was introduced and simulated. In fact, instead of adopting a unique radio access technology selection approach, the hybrid approach allows the use of a distributed solution with periodic system intervention to adjust the client association profile. This hybrid approach is a compromise between the two generic approaches (centralized and distributed approaches). It can improve the overall system performance (and the quality of service perceived by the clients. This solution will also induce additional processing costs and calculation charges due to the use of an exhaustive search at each intervention. It also reduces the signaling load by reducing the number of required handovers.

Operator's choice remains the key factor to monitor the system performance, to reduce costs experienced by mobile users, to reduce resource consumption and to make the most of those available. This choice is governed by several parameters which are mainly the characteristics of the system and the clients such as: the client arrival rate to the system, their session durations, the distribution of their peak rates, etc.

\section{REFERENCES}

[1] P. Lescuyer and T. Lucidarme, Evolved Packet Systems (EPS), John Wiley \& Sons, 2008.

[2] S. G. Matthew, 802.11 Wireless Networks: The Definitive Guide, O'Reilly, 2002.

[3] H. Labiod, H. Afifi, C. De Santis, Wi-Fi, Bluetooth, Zigbee and WiMAX, Springer, 2007.

[4] E. Hossain, Heterogeneous Wireless Access Networks Architectures and Protocols, Springer, 2008.

[5] M. Coupechoux, J.-M. Kelif, P. Godlewski, "Network Controlled Joint Radio Resource Management for Heterogeneous Networks," in Vehicular Technology Conf., 2008.

[6] J. Antoniou et al., "A Game Theoretic Approach for Network Selection," Univ. of Cyprus, Tech. Rep. TR-08-5, 2008.

[7] J. Antoniou and A. Pitsillides, "4G Converged Environment: Modeling Network Selection as a Game," in $16^{\text {th }}$ IST Mobile and Wireless Communications Summit, 2007, pp. 1-5.

[8] D. Kumar, E. Altman, J.M. Kelif, "User-Network Association in an 802.11 WLAN \& 3G UMTS Hybrid Cell: Individual Optimality," in Sarnoff Symp., USA, 2007.

[9] K. Khawam, M. Ibrahim, J. Cohen, S. Lahoud, S. Tohme, "Individual vs. Global Radio Resource Management in a Hybrid Broadband Network," in Int. Conf. Communications, Kyoto, Japan, 2011.

[10] A. Al Sabbagh, R. Braun, M. Abolhasan, "A Power Efficient RAT Selection Algorithm for Heterogeneous Wireless Networks," in Int. Symp. Communications and Information Technologies, 2012.

[11] F. Moety, M. Ibrahim, S. Lahoud, K. Khawam, "Distributed Heuristic Algorithms for RAT Selection in Wireless Heterogeneous Networks," in Wireless Communications and Networking Conf., Paris, France, 2012.

[12] V.Gazis, N. Alonistioti, L. Merakos, "Toward a Generic 'Always Best Connected' Capability in Integrated WLAN/UMTS Cellular Mobile Networks (and Beyond)," in IEEE Wireless Communications, 2005, pp. 20-29.

[13] M. Heusse, F. Rousseau, G. Berger-Sabbatel, A. Duda, "Performance Anomaly of $802.11 \mathrm{~b}, "$ in $22^{\text {nd }}$ Annu. Joint Conf. IEEE Computer and Communications, 2003, pp. 836-843. 